\title{
Analysis of the algorithm of the dual spectrum feature extraction of communication radio station
}

\author{
Wenle Yuan, Xuanmin Lu, Wensheng Luo, Jinjie Cao
}

School of Electronics and Information, Northwestern Polytechnical University, Xi'an, 710072, China

\begin{abstract}
In order to study the characteristics of double spectrum radio signals, based on higher order statistics and the double spectrum definition and properties, using two kinds of direct and indirect bispectrum feature extraction algorithm, and two kinds of radio signal bispectrum estimation is simulated. Therefore, it is concluded that the higher order statistics to inhibit Gauss process.
\end{abstract}

Keywords: Double spectrum; Higher-order Statistics; Fourier; Feature extraction; Pattern identification.

\section{Introduction}

Fingerprint recognition of communication signals includes three processes: preprocessing, feature extraction and classification. This paper mainly studies on the same model, the recognition method of the same batch of individual stations, including signal characteristics extraction for communication signals. The fine characteristics of the individual, this kind of method can be used in individual identification source of cooperation target. To identify the transient characteristics of the different kinds of radio communication in $25 \mathrm{~dB}$ environment under the condition of signal-to-noise ratio.

\section{Pattern Recognition}

Pattern recognition is according to the characteristics or attributes of the research object, the use of machine system with computer as the center, using some analysis algorithm finds its category system should enable the classification result as much as possible in line with the facts. The principle of pattern recognition system and recognition process as shown in Figure 1:

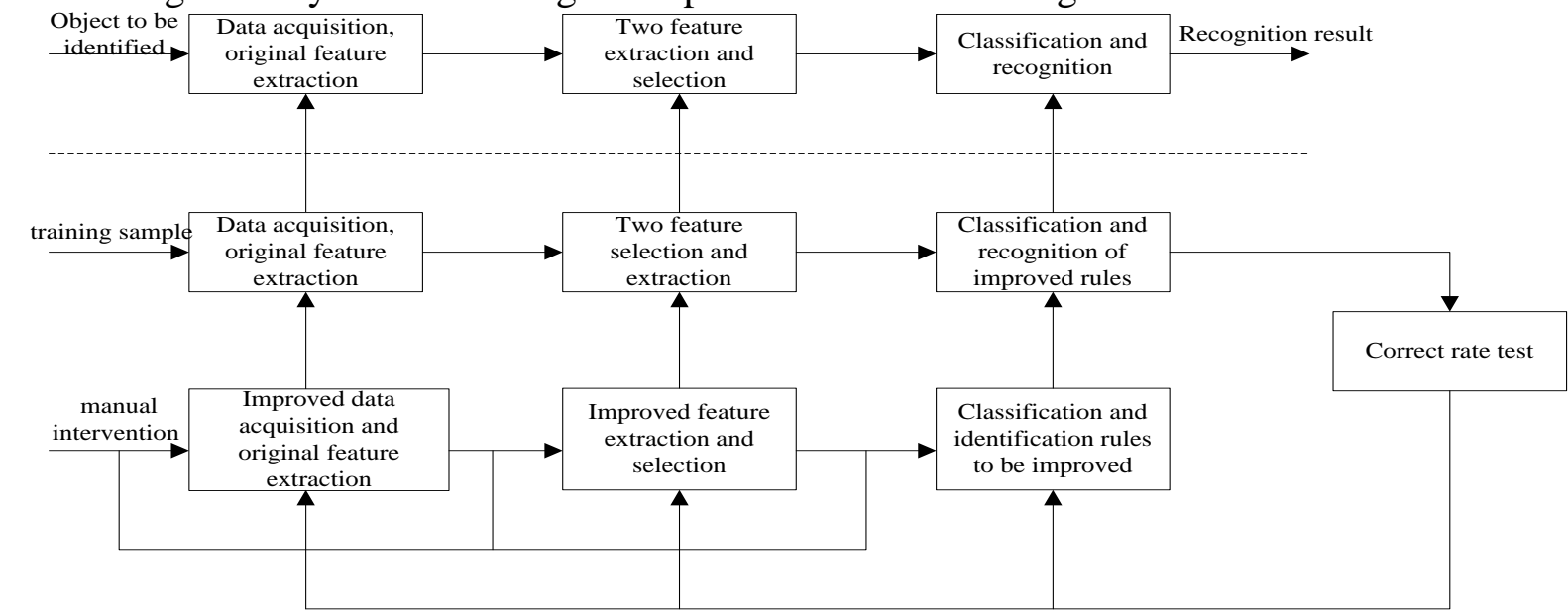

Figure 1 pattern recognition system and recognition process schematic

The basic idea of recognition method is based on the feature extraction algorithm to obtain the object is assigned a category with the identification mark, pattern recognition process from the information level, form conversion terms, by physical analysis of spatial objects through feature extraction into feature space model, and then through the classification of categories into the output space.

Fingerprint recognition of communication signals is essentially a problem of pattern recognition, through a mechanism to imitate human, realize the classification of objects, is a basic task in the field of pattern recognition. Design, decision-making is to use the designed classifier to deal with the analysis of the object of classification. Classification system mainly consists of 4 parts: information 
acquisition, data preprocessing, feature extraction and selection, classification and decision-making, as shown in Figure 2.

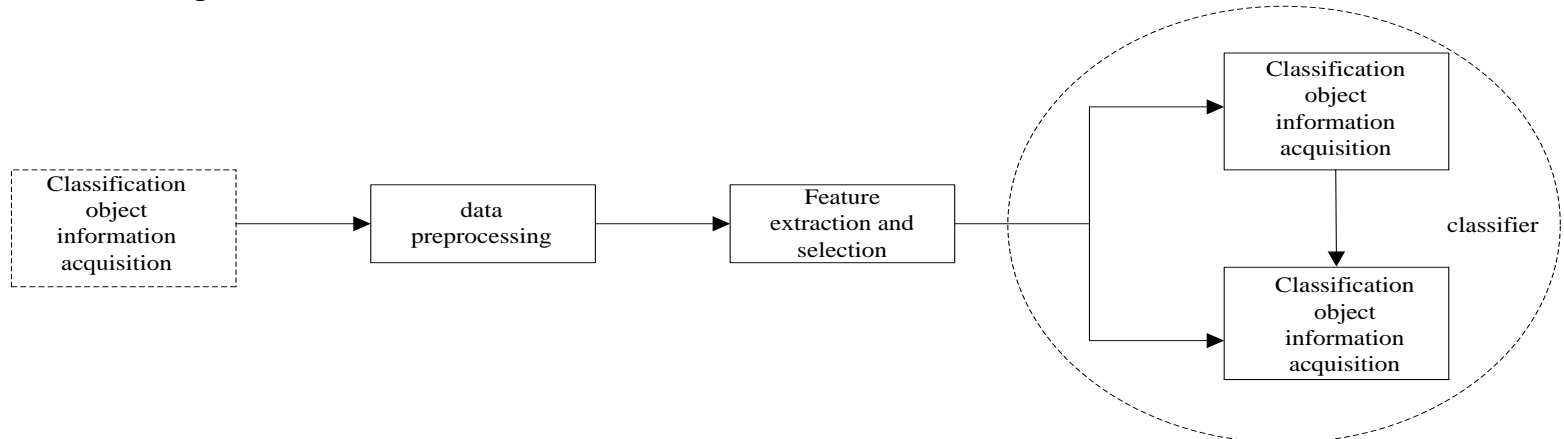

Figure 2 communication radiation source individual identification principle block diagram

\section{Higher order statistics theory}

\subsection{Characteristic function of random variable}

Define 1 order random variables $\mathrm{X}$ probability density function $f(x)$, the characteristic function is defined as integral.

$$
\Phi(\omega)=\int_{-\infty}^{+\infty} f(x) e^{j w x} d x=E\left[e^{j w x}\right]
$$

The characteristic function is density $f(x)$ the Fu Liye transform.Because $f(x) \geq 0$, therefore, the origin of the characteristic function has the maximum value.

$|\Phi[\omega]| \leq \Phi(0)=1$

The characteristic function is also called the first characteristic function.

Second characteristic function $\varphi(\omega)$ is defined as

$\varphi(\omega)=\ln [\Phi(\omega)]$

Define 2 orders $\mathrm{X}=\left[x_{1}, x_{2}, \ldots, x_{n}\right]^{\mathrm{T}}$ is a random variable, Joint probability density $f\left(x_{1}, x_{2}, \ldots, x_{n}\right)$, the characteristic function is defined as integral

$$
\Phi\left(\omega_{1}, \omega_{2}, \ldots, \omega_{n}\right)=\int_{-\infty-\infty}^{+\infty+\infty} f\left(x_{1}, x_{2}, \ldots, x_{n}\right) e^{j\left(\omega_{1} x_{1}+\omega_{2} x_{2}+\ldots+\omega_{n} x_{n}\right)} d x_{1} d x_{2} \ldots d x_{n}
$$

At present $\Phi\left(\omega_{1}, \omega_{2}, \ldots, \omega_{n}\right)=E\left[e^{j\left(\omega_{1} x_{1}+\omega_{2} x_{2}+\ldots+\omega_{n} x_{n}\right)}\right]$

The second characteristic function of random variables is defined as

$\varphi\left(\omega_{1}, \omega_{2}, \ldots, \omega_{n}\right)=\ln \left[\Phi\left(\omega_{1}, \omega_{2}, \ldots, \omega_{n}\right)\right]$

\subsection{Higher order moment and higher order}

Define 6 High order moment $m_{k x}\left(\tau_{1}, \tau_{2}, \ldots, \tau_{k-1}\right)$ is absolutely available:

$$
\sum_{\tau_{1}=-\infty}^{+\infty} \ldots \sum_{\tau_{k-1}}^{+\infty}\left|m_{k x}\left(\tau_{1} \tau_{2}, \ldots, \tau_{k-1}\right)\right|<\infty
$$

$k$ the order moment spectrum is defined as $k$ moment of order $k-1$ dimensional Fourier transformation, at present

$$
M_{k x}\left(\omega_{1}, \omega_{2}, \ldots, \omega_{k-1}\right)=\sum_{\tau_{1}=-\infty}^{+\infty} \ldots \sum_{\tau_{k-1}=-\infty}^{+\infty} m_{k x}\left(\tau_{1}, \tau_{2}, \ldots, \tau_{k-1}\right) \exp \left[-j \sum_{i=1}^{k-1} \omega_{i} \tau_{i}\right]
$$

Define 7 High order moment $c_{k x}\left(\tau_{1} \tau_{2}, \ldots, \tau_{k-1}\right)$ is absolutely available:

$$
\sum_{\tau_{1}=-\infty}^{+\infty} \ldots \sum_{\tau_{k-1}}^{+\infty}\left|c_{k x}\left(\tau_{1}, \tau_{2}, \ldots, \tau_{k-1}\right)\right|<\infty
$$

$k$ the order moment spectrum is defined as $k$ moment of order $k-1$ dimensional Fourier transformation, at present 


$$
S_{k x}\left(\omega_{1}, \omega_{2}, \ldots, \omega_{k-1}\right)=\sum_{\tau_{1}=-\infty}^{+\infty} \ldots \sum_{\tau_{k-1}=-\infty}^{+\infty} c_{k x}\left(\tau_{1}, \tau_{2}, \ldots, \tau_{k-1}\right) \exp \left[-j \sum_{i=1}^{k-1} \omega_{i} \tau_{i}\right]
$$

The most common higher order spectra are:

Three order spectrum (double spectrum):

$$
B_{x}\left(\omega_{1}, \omega_{2}\right)=\sum_{\tau_{1}=-\infty}^{\infty} \sum_{\tau_{2}=-\infty}^{\infty} c_{3 x}\left(\tau_{1}, \tau_{2}\right) e^{-j\left(\omega_{1} \tau_{1}+\omega_{2} \tau_{2}\right)}
$$

Four order spectra (three spectra):

$$
\mathrm{T}_{x}\left(\omega_{1}, \omega_{2}, \omega_{3}\right)=\sum_{\tau_{1}=-\infty}^{\infty} \sum_{\tau_{2}=-\infty}^{\infty} \sum_{\tau_{3}=-\infty}^{\infty} c_{4 x}\left(\tau_{1}, \tau_{2}, \tau_{3}\right) e^{-j\left(\omega_{1} \tau_{1}+\omega_{2} \tau_{2}+\omega_{3} \tau_{3}\right)}
$$

Define $8\{x(n), n=0, \pm 1, \pm 2, \ldots\}$ is a deterministic signal with finite energy, then its Fourier transform, energy spectrum, double spectrum and three spectrum are defined as follows:

$$
\begin{array}{ll}
\text { Fourier transform } & X(\omega)=\sum_{k=-\infty} x(k) e^{-j \omega k} \\
\text { Energy spectrum } & \mathrm{P}_{x}\left(\omega_{1}, \omega_{2}\right)=X(\omega) X^{*}(\omega) \\
\text { Double spectrum } & \mathrm{B}_{x}\left(\omega_{1}, \omega_{2}\right)=X\left(\omega_{1}\right) X\left(\omega_{2}\right) X^{*}\left(\omega_{1}+\omega_{2}\right) \\
\text { Three spectrum } & \mathrm{T}_{x}\left(\omega_{1}, \omega_{2}, \omega_{3}\right)=X\left(\omega_{1}\right) X\left(\omega_{2}\right) X\left(\omega_{3}\right) X^{*}\left(\omega_{1}+\omega_{2}\right)
\end{array}
$$

\subsection{The properties and algorithms of the double spectrum}

Multi spectral static signal sequence $x(n)$ of $k$ the order of the cumulative quantity is defined as follows:

$$
m_{k x}\left(\tau_{1} \tau_{2}, \ldots, \tau_{k-1}\right)=E\left\{x(n), x\left(n+\tau_{1}\right), \ldots, x\left(n+\tau_{k-1}\right)\right\}
$$

$k$ the order power spectrum is defined as follows:

$$
M_{k x}\left(\omega_{1}, \omega_{2}, \ldots, \omega_{k-1}\right)=\sum_{\tau_{1}=-\infty}^{+\infty} \ldots \sum_{\tau_{k-1}=-\infty}^{+\infty} m_{k x}\left(\tau_{1} \tau_{2}, \ldots, \tau_{k-1}\right) \exp \left[-j \sum_{i=1}^{k-1} \omega_{i} \tau_{i}\right]
$$

$k=3$ at the time of the multi spectrum called power spectrum or double spectrum, the lowest order, the processing method is the most simple, contains the power spectrum of the phase information, and can effectively suppress the Gauss noise, is the focus of higher order spectral research.

Among, "** "complex conjugate, double spectrum $\mathrm{B}_{x}\left(\omega_{1}, \omega_{2}\right)$ the symmetric line is shown in Figure 3. $\omega_{2}=0$ 和 $\omega_{1}=\omega_{2}, 2 \omega_{1}=-\omega_{2}, 2 \omega_{2}=-\omega_{1}, \omega_{1}=-\omega_{2}, \omega_{1}=0$. These lines divide the definition region into 12 segments, draw area $C=\left\{\omega_{1}, \omega_{2}: 0 \leq \omega_{1}, \omega_{2} \leq \omega_{1}\right\}$ is dual spectrum $\mathrm{B}_{x}\left(\omega_{1}, \omega_{2}\right)$ in $\left(\omega_{1}, \omega_{2}\right)$ within the plane of the main domain, the symmetric nature of the double spectrum is known, as long as the main domain of the dual spectrum, it can completely describe all of the dual spectrum.

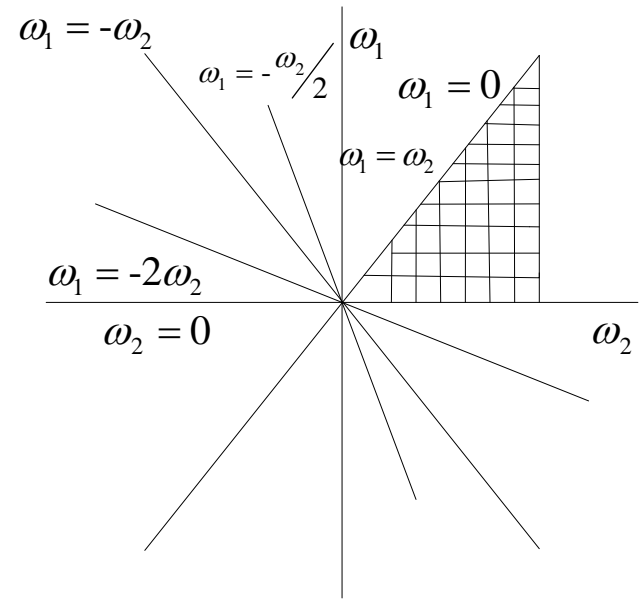

Figure 3 the double spectrum symmetry lines and the main fields of continuous time signals 
(1) Direct method

Steps are as follows:

The data segment is given $\mathrm{K}$ segment, each section contains $\mathrm{M}$ observation samples, $\mathrm{N}=\mathrm{KM}$, and the average value of each segment is subtracted from the data. If necessary, zero is added to the data in order to meet the requirements of the general length $m$ of the FFT.

1) Calculated DFT coefficient

$$
\mathrm{Y}^{(1)}(\lambda)=\frac{1}{\mathrm{M}} \sum_{n=0}^{\mathrm{M}-1} y^{(i)}(n) \exp (-2 j 2 \pi n \lambda / \mathrm{M}), \lambda=0,1, \ldots, \mathrm{M} / 2 ; i=1,2, \ldots, \mathrm{K}
$$

Among $\left\{y^{(i)}(n), n=1,2, \ldots, \mathrm{M}-1\right\}$ is the first $i$ data segment.

2) Calculation of the DFT coefficient of the three weight correlation:

$$
\hat{b}\left(\lambda_{1}, \lambda_{2}\right)=\frac{1}{\Delta_{0}^{2}} \sum_{k_{1}=-L_{1}}^{L_{1}} \sum_{k_{2}=-L_{1}}^{L_{1}} \mathrm{Y}^{(i)}\left(\lambda_{1}+k_{1}\right) \mathrm{Y}^{i}\left(\lambda_{2}+k_{2}\right) \times \mathrm{Y}^{(i)}\left(-\lambda_{1}-\lambda_{2}-k_{1}-k_{2}\right)
$$

Among $0 \leq \lambda_{2} \leq \lambda_{1}, \lambda_{1}+\lambda_{2} \leq f_{s} / 2, \mathrm{~N}_{0}, L_{1}$ should meet $\mathrm{M}=\left(2 L_{1}+1\right) \mathrm{N}_{0}$ value.

3) The double spectrum of the given data is given by the average value of the $\mathrm{K}$ segment.

$\hat{\mathrm{B}_{D}}\left(\overline{\omega_{1}},-\overline{\omega_{2}}\right)=\frac{1}{\mathrm{~K}} \sum_{i=1}^{\mathrm{K}} \hat{b_{i}}\left(\overline{-}-\overline{\omega_{1}}, \overline{\omega_{2}}\right)$

Among

$\overline{\omega_{1}}=\left(\frac{2 \pi f_{s}}{\mathrm{~N}_{0}}\right) \lambda_{1}, \overline{\omega_{2}}=\left(\frac{2 \pi f_{s}}{\mathrm{~N}_{0}}\right) \lambda_{2}$

(2) Indirect method

Steps are as follows:

1) Will data $x(1), x(2), \ldots, x(n)$ divided into $\mathrm{k}$ segments, each with $\mathrm{m}$ samples, and subtract the respective mean.

$\left\{x^{(i)}(n), n=1,2, \ldots, M-1\right\}$ is the first $i$ data segment, an estimated three order cumulant segments:

$c^{(i)}(l, k)=\frac{1}{\mathrm{M}} \sum_{t=\mathrm{M}}^{\mathrm{M}_{2}} x^{(i)}(t) x^{(i)}(t+1) x^{(i)}(t+k), i=1,2, \ldots, k$

Type

$$
\begin{aligned}
& \mathrm{M}_{1}=\max (0,-l,-k) \\
& \mathrm{M}_{2}=\min (\mathrm{M}-1, \mathrm{M}-1-l, \mathrm{M}-1-k)
\end{aligned}
$$

2) The average of the three order of all segments is estimated as the three order of the whole observation data set:

$$
\hat{c}(k, 1)=\frac{1}{\mathrm{~K}} \sum_{i=1}^{\mathrm{K}} c^{(i)}(l, k)
$$

3) Generation of double spectrum estimation:

$$
\hat{\mathrm{B}_{\mathrm{IN}}}\left(\overline{\omega_{1}}, \overline{\omega_{2}}\right)=\sum_{l=-L k=-L}^{L} \sum^{L} \hat{c}(l, k) \bar{\omega}(l, k) \exp \left\{-j\left(\overline{\omega_{1}} l+\overline{\omega_{2}} k\right)\right\}
$$

Type $L<\mathrm{M}-1$, but $\omega(1, k)$ generation of double spectrum estimation:

Due to the symmetry of the (21) use of the three order of the cumulative amount, and in the (23) in the use of the symmetry of the double spectrum, the calculation of indirect algorithm can be greatly decreased. 


\section{Analysis of the characteristics of communication signal}

The biggest advantage of higher order statistics can suppress Gauss noise, can provide useful information from signal noise, and effectively improve the recognition rate. The linear assumption, and the traditional Gauss noise signal, stationary or local stationarity, the actual signal noise is a nonlinear, non-Gauss and smooth signal. And the higher order statistics method is a powerful tool of communication signals of three non-noise characteristics. The high order spectrum can not only restrain the Gauss noise, but also reflect the combined signal phase and phase information. The lotus type with two kinds of signal as an example, the bispectrum estimation, such as figure 4:

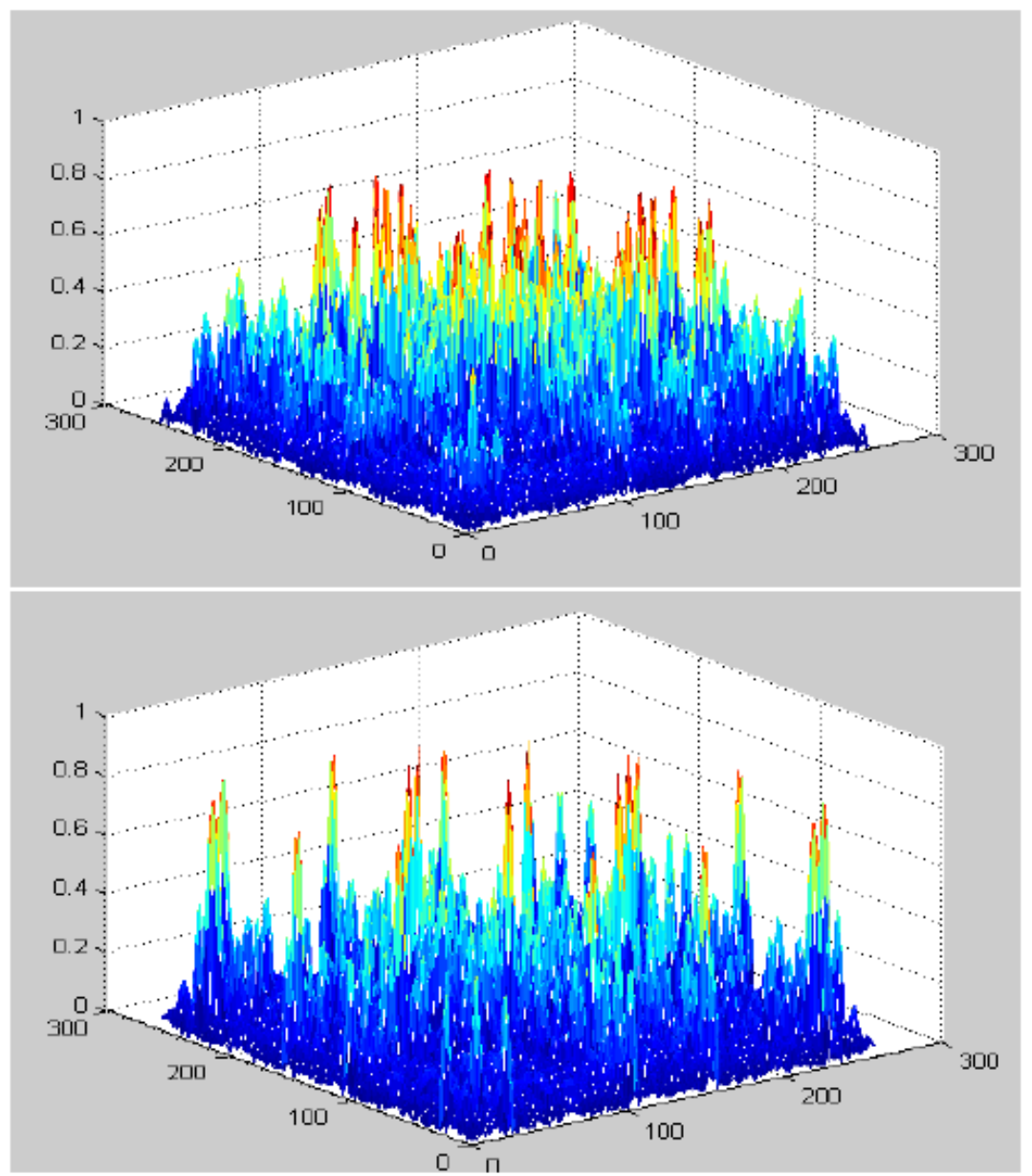

Figure 4 two kinds of radio signals of the dual spectrum

This section of two kinds of radio signals in the double spectrum estimation, noise two signals are non-Gauss components. From the above, 4 different radio signal spectrum can be seen double difference signal noise in the continuous spectrum and intensity and so on.

\section{Summary}

This paper starts from the characteristic function of random variables, random variables of higher-order moments and cumulants and the corresponding spectrum gives the definition, introduces the Gauss process of higher-order moments and cumulants and some basic properties. Finally, considering the needs of feature extraction, this chapter focuses on the nature of the double spectrum and two kinds of commonly used algorithms, and simulation analysis of double spectrum communication signal noise.

\section{Acknowledgements}

Northwestern Polytechnical University graduate creative new seed fund. 


\section{References}

[1] J. Toonstra, W. Kinsner. A Radio Transmitter Fingerprinting System ODO-1 [C]. Canadian Conference on Electrical and Computer Engineering, 1996, (1): 60-63.

[2] D. Shaw, W. Kinsner. Multifractal modelling of radio transmitter transients for classification [J]. IEEE Proceedings, WESCANEX 97: Communications, Power and Computing, IEEE, 22-23 May 1997, 306-312.

[3] Xianbin Wang, Yiyan Wu, B. Caron. Transmitter identification using embedded spread spectrum sequences [C]. Proc. International Conference on Communication Technology, ICCT 2003, (2): $1517-1523$.

[4] V. N. VaPnik. Statistical Learning Theory [M]. New York Wiley, 1998.

[5] Cortes C, Vapnik V. Support Vector Networks. Machine Learning, 1995, 20(3): 273-297.

[6] N.Cristianini and J shawe-Taylao. An Introduction to support vector machines [M]. Cambridge: Cambridge University press, 2000.

[7] V. N. Vpanik and A. Y. Chervoenkis. Theory of Pattern Recognition. Moscow [M]. Russia Nauka, 1974.

[8] John Shawe-Taylor. Kernel Methods for Pattern Analysis [M]. University of Southampton: Cambridge University Press, 2004.

[9] Shuhua Xu, Benxiong Huang, Zhengguang Xu, Yuchun Huang. A NEW FEATURE VECTOR USINGLOCAL FOR IDENTIFYING RADIO TRANSMITTERS [J]. Department of Electronics and Information, Huazhong University of Sci, and Tech, Wuhan, 2007:176-178. 